\title{
Delineation of the ADULT syndrome phenotype due to arginine 298 mutations of the $p 63$ gene
}

\author{
Tuula Rinne ${ }^{1}$, Emanuela Spadoni ${ }^{2}$, Klaus W Kjaer ${ }^{3}$, Cesare Danesino ${ }^{2}$, Daniela Larizza ${ }^{4}$, \\ Marianne Kock $^{5}$, Kirsi Huoponen ${ }^{6}$, Marja-Liisa Savontaus ${ }^{6}$, Markku Aaltonen ${ }^{7}$, Pascal Duijf ${ }^{1}$, \\ Han G Brunner ${ }^{1}$, Maila Penttinen ${ }^{8}$ and Hans van Bokhoven*,1
}

\begin{abstract}
${ }^{1}$ Department of Human Genetics, Radboud University Nijmegen Medical Centre, Nijmegen, The Netherlands; ${ }^{2}$ Biologia Generale e Genetica Medica, University of Pavia and IRCCS San Matteo, Pavia, Italy; ${ }^{3}$ Wilhelm Johannsen Centre for Functional Genome Research, Department of Medical Biochemistry and Genetics, University of Copenhagen, Copenhagen, Denmark; ${ }^{4}$ Department of Paediatrics, IRCCS San Matteo, Pavia, Italy; ${ }^{5}$ Department of Orthodontics, University of Copenhagen, Copenhagen, Denmark; ${ }^{6}$ Department of Medical Genetics, University of Turku, Turku, Finland; ${ }^{7}$ Department of Pathology, University of Turku, Turku, Finland; ${ }^{8}$ Clinical Genetics Unit, Department of Pediatrics, Turku University Central Hospital, Turku, Finland
\end{abstract}

The ADULT syndrome (Acro-Dermato-Ungual-Lacrimal-Tooth, OMIM 103285) is a rare ectodermal dysplasia associated with limb malformations and caused by heterozygous mutations in p63. ADULT syndrome has clinical overlap with other $p 63$ mutation syndromes, such as EEC (OMIM 604292), LMS (OMIM 603543), AEC (106260), RHS (129400) and SHFM4 (605289). ADULT syndrome characteristics are ectrodactyly, ectodermal dysplasia, mammary gland hypoplasia and normal lip and palate. The latter findings allow differentiation from EEC syndrome. LMS differs by milder ectodermal involvement. Here, we report three new unrelated ADULT syndrome families, all with mutations of arginine 298. On basis of 16 patients in five families with R298 mutation, we delineate the ADULT syndrome phenotype. In addition, we have documented a gain-of-function effect on the dNp63gamma isoform caused by this mutation. We discuss the possible relevance of oral squamous cell carcinoma in one patient, who carries this p63 germline mutation.

European Journal of Human Genetics (2006) 14, 904-910. doi:10.1038/sj.ejhg.5201640; published online 17 May 2006

Keywords: ADULT; ectodermal dysplasia; ectrodactyly; $p 63$

\section{Introduction}

Various combinations of limb anomalies, ectodermal dysplasias and orofacial clefts characterize heterozygous mutations in the transcription factor gene $p 63$. The prototype $p 63$ mutation syndrome is EEC syndrome, which is characterized by ectrodactyly, ectodermal dysplasia and

*Correspondence: Dr H van Bokhoven, Department of Human Genetics 855, Radboud University Nijmegen Medical Centre, PO Box 9101, 6500 HB Nijmegen, The Netherlands.

Tel: + 3124361 6696; Fax: + 3124366 8752;

E-mail: h.vanbokhoven@antrg.umcn.nl

Received 19 January 2006; revised 22 March 2006; accepted 23 March 2006; published online 17 May 2006 cleft lip/palate (OMIM 604292). Ectrodactyly, a severe median cleft in hands and feet is often associated with syndactyly in these patients, but syndactyly can also be the sole limb defect. Ectodermal dysplasia can be found in various combinations of sparse hair, dry skin, hypodontia, dysplastic nails and alterations in sebaceous glands, mammary glands and nipples. Orofacial clefting is the third hallmark of the EEC syndrome and usually manifests as cleft lip with cleft palate. Symptoms in the other five inherited syndromes with $p 63$ mutations are overlapping, but each of these syndromes has its own characteristic combination of phenotypic features. For instance, ankyloblepharon is almost limited to the Hay-Wells syndrome 
(ankyloblepharon-ectodermal defects-cleft lip/palate, AEC, OMIM 106260). Mammary gland hypoplasia and nipple hypoplasia are frequent findings in limb mammary syndrome (LMS, OMIM 603543) and in acro-dermatoungual-lacrimal-tooth syndrome (ADULT, OMIM 103285) but are rare in other p63-related conditions. ${ }^{1}$ ADULT syndrome is further distinguished from other p63 syndromes by absence of orofacial clefting and by prominent ectodermal signs. Abnormal hair or skin abnormalities have not been reported among LMS patients. Facial clefting in LMS is of another type than that in EEC syndrome: cleft palate only, whereas no facial clefts have been found among reported ADULT families. ${ }^{2-6}$ In one family, extensive freckling has been reported to be a typical characteristic of ADULT syndrome, and this was also mildly present in two further ADULT syndrome patients. ${ }^{2,4,7}$

The causative gene in all these disorders, $p 63$, is crucial during embryonic development, mostly in the development of limbs and other ectodermal derived tissues. The pattern of mutations in six different p63-related syndromes shows genotype-phenotype correlations. The most pronounced genotype-phenotype correlation is in the EEC and Hay-Wells syndromes. All EEC missense mutations are clustered in the DNA binding domain and disrupt binding to DNA. ${ }^{8}$ In contrast, all missense mutations reported for Hay-Wells patients are in the Sterile-Alpha-Motif domain, and have been shown to disrupt interaction with other proteins. ${ }^{9}$ LMS and ADULT syndrome have their own unique mutated amino-acid residues. In the original LMS family a point mutation was found in the coding region of the second transactivation domain $(\mathrm{G} 76 \mathrm{~W}) .^{3}$ Only two amino-acid residues are known to be mutated among ADULT syndrome patients: asparagine 6 , which is in the putative second transactivation domain and arginine 298, which locates in the DNA binding domain. ${ }^{4,5,7}$ Although R298 is in the DNA binding domain, it is functionally different from the EEC mutations, because its substitution by glutamine does not lead to a loss of DNA binding, but instead to a gain of transactivation activity of the deltaNp63gamma isoform. ${ }^{6}$ Here, we report three further ADULT syndrome families with R298 mutations, either a R298Q or R298G. We discuss the consistent phenotypic features associated with these gain-of-function mutations.

\section{Materials and methods Patients and families}

In a small Finnish family three family members, one in each generation, present with an EEC-like disorder. Their limb malformations vary from severe four-limb ectrodactyly and syndactyly in the oldest person (Figure $1 \mathrm{a}-\mathrm{b}$ ) to unilateral syndactyly in the child of the third generation. All patients have a similar pattern of malformations in
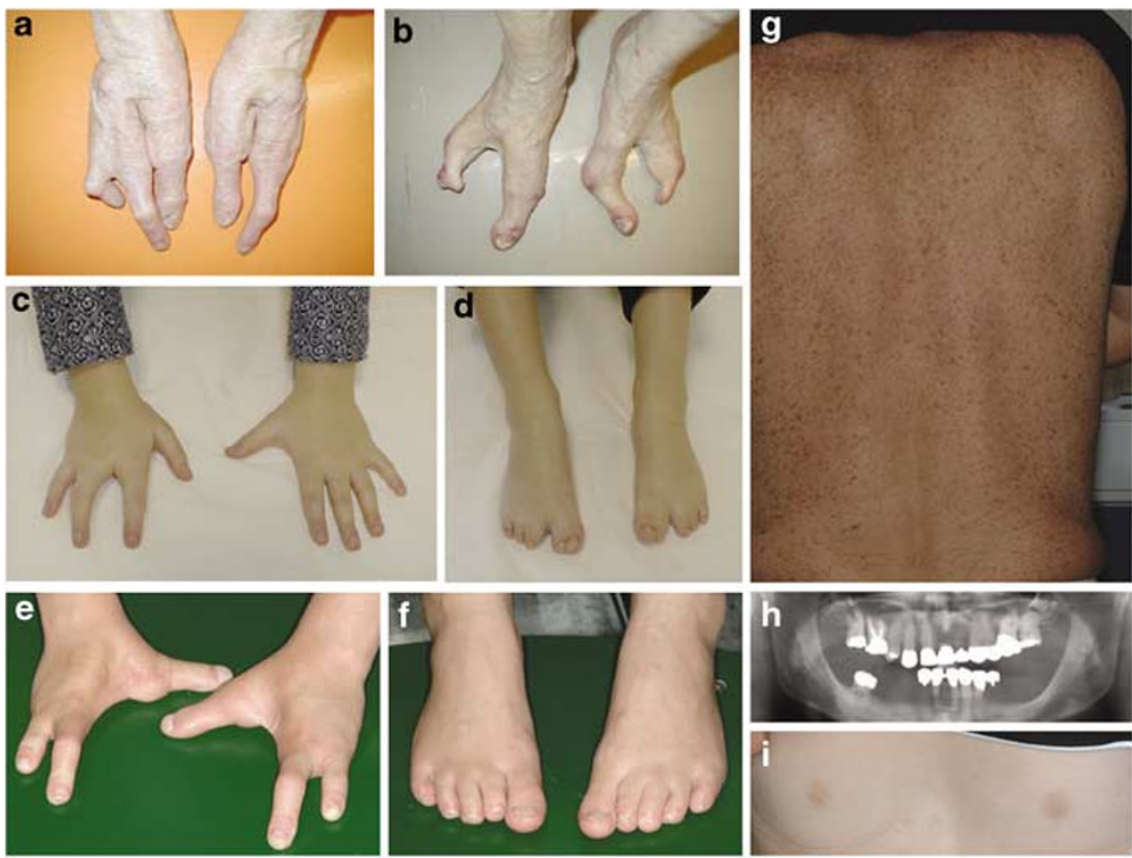

Figure 1 Limb and ectodermal phenotype in ADULT syndrome patients with a R298 mutation. Limb malformations in R298 patients can vary much. (a, b) Deep median cleft and syndactyly in the limbs of the Finnish ADULT syndrome patient (I/1) with R298G mutation. (c, d) Mildly affected limbs in the Italian patient (II/1) with R298Q mutation. (e, f). Severely affected hands and nonaffected feet of Danish ADULT patient (II/1) with R298Q mutation. (g) Excessive freckling in Danish patient (I/1). (h) Hypodontia and loss of permanent teeth in Danish patient (I/1). (i) Hypoplastic nipples in Danish patient (II/1). 
ectodermal derived tissues as described in the original German ADULT syndrome family. ${ }^{2,4}$ Atrophic, dry skin is present, but freckling was not seen. Breast hypoplasia is found in adult female patients. The 9-year-old patient has widely spaced nipples and congenital lacrimal duct obstruction. All affected individuals have very blond, thin and sparse hair. Nails are reported to be dysplastic, and one or several teeth were lacking. A malignant tumour in the mouth of one of the patients was diagnosed at age 60 years. Histological examination confirmed a squamous cell carcinoma (SCC). Otherwise the medical history is unremarkable for all patients.

In a small Italian family, mother and son have symptoms similar to those described above for the Finnish family, and previously reported for a German family. ${ }^{2,4}$ The son has absence of the middle finger in his right hand and cutaneous syndactyly in both feet together with right foot polydactyly (Figure 1c-d). His mother has normal limbs. Both affected individuals have lightly pigmented, dry and very photosensitive skin. Dermatitis has occurred in both individuals. Both mother and son have dysplastic nails with pits. The mother has oligodontia (dysplastic and hypoplastic teeth), whereas the son has only small teeth. The mother also has breast hypoplasia and absence of the right nipple, and the son has hypoplastic nipples. The son has tear duct obstruction and his mother absence of tears and chronic conjunctivitis, probably due to obstructed tear ducts. The mother was recently diagnosed with autoimmune thyroiditis and paroxysmal supraventricular tachycardia. The maternal grandfather of the son died at the age of 36 years from a clear-cell renal carcinoma. He had lightly pigmented hair and skin, and he was highly photosensitive, like his family members. Otherwise, no EEC-like symptoms are reported for the grandfather.

In a Danish family, father and daughter have symptoms similar to those described above for the Finnish and the Italian family. Ectrodactyly and syndactyly is present in the hands and one foot of the father and ectrodactyly is present in the hands of the daughter (Figure $1 \mathrm{e}-\mathrm{f}$ ). Both father and daughter have nail dystrophy in all nails. Their skin is dry and thin, and excessive freckling of the entire body surface is present (Figure 1g). Father and daughter both have hypodontia and dysplastic teeth (Figure $1 \mathrm{~h}$ ). The daughter has hypoplastic nipples (Figure 1i). The hair is unaffected in this family. The father has blocked tear ducts, as does his daughter, who had surgery when she was a child. There is no orofacial clefting in these patients.

\section{Mutation analysis}

From the Finnish family blood samples were obtained from three patients and the proband's healthy sister after a written informed consent was obtained. Genomic DNA was extracted using a Nucleon Extraction \& Purification Kit (Amersham Biosciences). The exons, which have been reported to contain most pathogenic mutations of $p 63$, were amplified by PCR and the products were purified by GFX $^{\mathrm{TM}}$ PCR DNA and Gel Band Purification Kit (Amersham Biosciences) and sequenced directly using an ABI PRISM ${ }^{\mathrm{TM}}$ 377 automated sequencer (Applied Biosystems). The observed sequence change was confirmed with a restriction fragment analysis using HaeIII enzyme (New England BioLabs). In total, 54 control persons were tested for the presence of the pathogenic mutation. Mutation analysis for the Italian family was performed as described by Duijf et $a l^{6}$.

From the Danish family blood samples were obtained from two affected and two nonaffected members after a written informed consent was obtained. Genomic DNA was extracted from peripheral blood samples using a standard salting-out method. All exons and exon-intron boundary regions were sequenced on both strands. Primers were removed by treatment with $1 \mathrm{U}$ shrimp alkaline phosphatase (USB, Cleveland, OH, USA) and $10 \mathrm{U}$ exonuclease I (New England Biolabs) followed by sequencing using the BigDye Terminator Kit (Applied Biosystems, Foster City, CA, USA) and analysed on an ABI 377 sequencer (Applied Biosystem).

\section{Functional analysis of R298G mutation}

We performed a transactivation assay to see whether the R298G mutation causes a gain-of-function effect for deltaNp63gamma isoform similar to the R298Q mutation. ${ }^{6}$ Mutagenesis and transactivation assays were performed as described previously ${ }^{6}$. Site-directed mutagenesis for introduction of the R298G mutation was performed with oligonucleotide 5'-cgcaagtcctgggcggacggtgctttgag-3' and its reverse complement as described previously. ${ }^{8}$

\section{Immunohistochemical staining}

A four-micrometer-thick section was cut from the formalin-fixed paraffin-embedded oral mucosa SCC sample. Immunohistochemical staining was performed using a TechMate500 immunostaining machine and a peroxidase/diaminobenzine (DAB) multilink detection kit (DAKO, Denmark). The section was stained with 4A4 monoclonal antibody (1:180) (a gift from Dr F McKeon, Harvard, USA) and counterstained with Mayer's haematoxylin.

\section{Results \\ Mutation analysis}

Mutation analysis of the $p 63$ gene was carried out by direct sequencing of p63 exons in the probands of the Finnish, the Italian and the Danish family. In the Italian and the Danish family a heterozygous transition from a guanine to an adenine was found at nucleotide position 893. This mutation is located at the beginning of exon 8 and causes a change from CGA to CAA at codon 298. This mutation is identical to one that was originally identified in the 
German ADULT syndrome family and predicts an amino acid change from arginine to glutamine (R298Q). ${ }^{6,10}$ Later this mutation was also reported by Chan et $a l^{7}$. In the Finnish family a novel $p 63$ mutation was found at the same codon 298: a heterozygous point mutation changing a cytosine to a guanine at nucleotide position 892 , which creates a change from CGA to GGA at codon 298 and a predicted amino-acid change from arginine to glycine (R298G) in the DNA binding domain. In total, 54 control samples were tested for the mutation with a restriction fragment analysis with the restriction enzyme HaeIII, which cleaves the wild-type amplicon, but not the mutated DNA fragment. None of the control samples or healthy family members carried the R298G mutation. Both mutations fully segregated with the phenotype in the three families.

\section{Transactivation studies}

According to the structural model of the DNA binding domain of p63, the arginine at position 298 does not locate close to binding DNA. ${ }^{6}$ Indeed, transactivation studies have established that the R298Q mutation does not impair transactivation activity as do EEC causing mutations. ${ }^{6,8}$ Instead, the deltaNp63gamma isoform carrying the R298Q mutation has highly increased transactivation activity as compared to its wild-type counterpart. To investigate whether this effect is specific for this aminoacid substitution, we decided to test the effect of the newly identified R298G mutation in transactivation assays. Functional analysis of the R298G mutation also yielded an exceptionally high transactivation activity, very similar to the activity, which was observed for the R298Q ADULT syndrome mutation (Figure 2). It thus appears that the functional consequences of these mutations are comparable.

\section{Immunohistochemical studies}

Immunohistochemical staining using the $4 \mathrm{~A} 4$ monoclonal antibody showed strong p63 accumulation in the undifferentiated SCC of the R298G-patient (Figure 3).

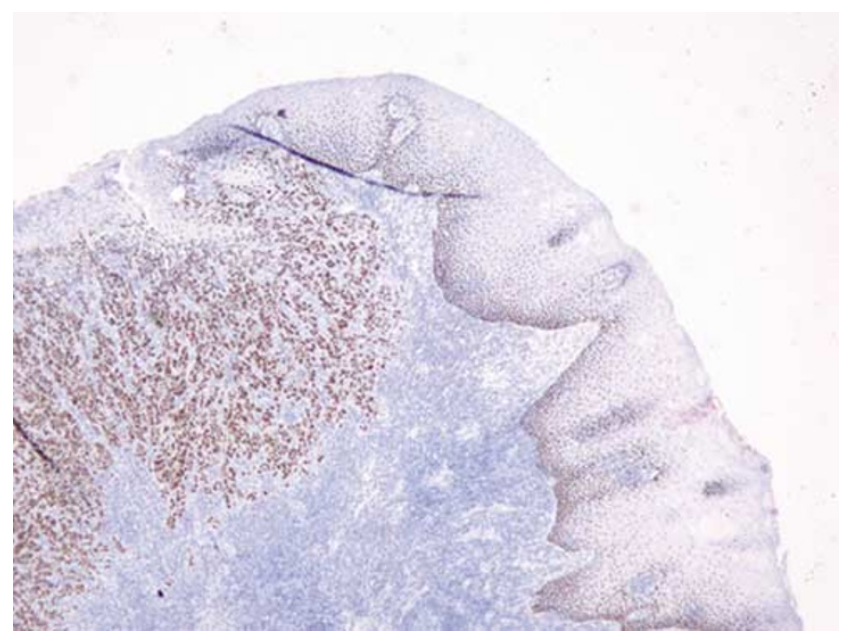

Figure 3 Undifferentiated oral SCC of the Finnish ADULT syndrome (R298G) patient. The SCC oral mucosa (on the left) shows strong p63 immunoreactivity, whereas the p63 expression in the healthy mucosa is strongest in the basal layer and gradually decreased in the suprabasal layers (on the right). (Magnification is $\times 200$ ).

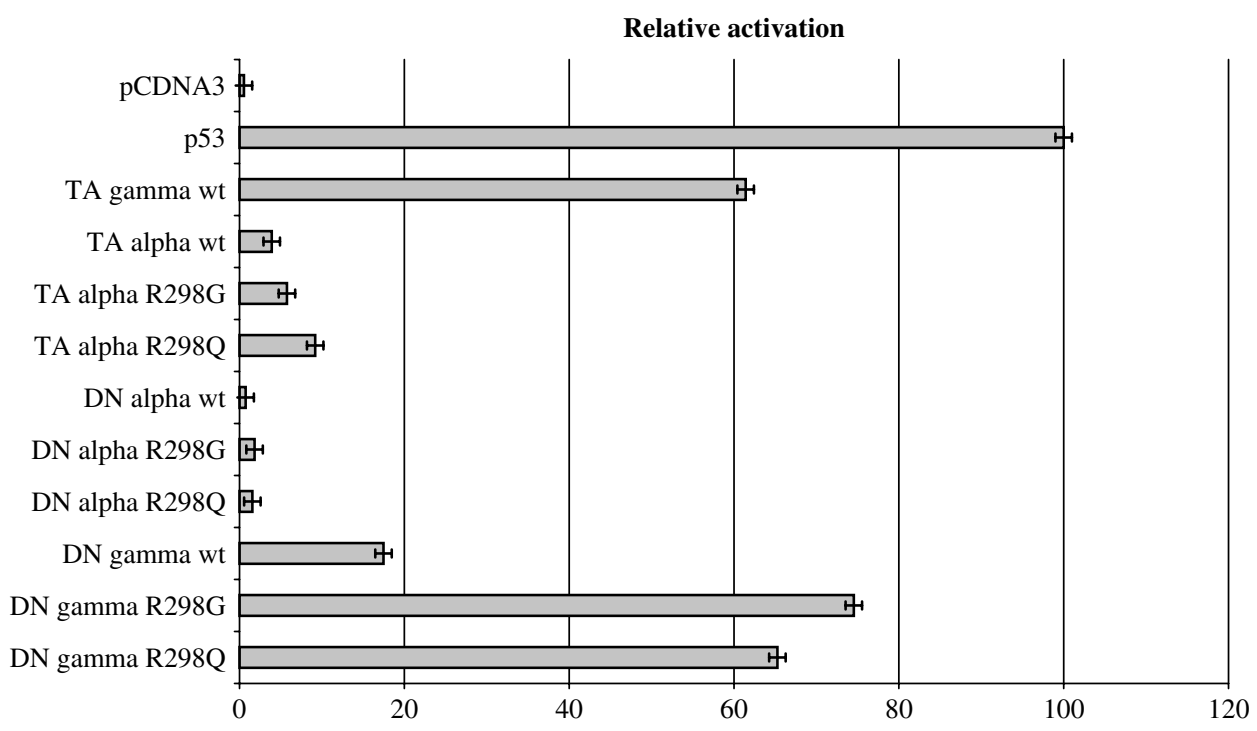

Figure 2 Transactivation of the wild-type p63, R298Q and R298G mutant isoforms. Transactivation assays are performed in extracts from SaOs-2 cells, which were transfected with wild type, R298Q or R298G mutant constructs. The relative transactivation activity is given with respect to the p53 expression vector. TAp63gamma wild type is the only isoform, which is known to be able to transactivate p53 response elements. Here, both R298Q and R298G mutations cause a gain of activation on dNp63gamma isoform, whereas the other mutated isoforms resemble the wild-type p63 behaviour. Transactivation activity data of the TAp63gamma R298Q/G mutation constructs is not shown. 


\section{Clinical evaluation of patients with a mutation at R298}

Having established that the R298G mutation has similar functional effects as the R298Q mutation, we next draw a comparison of the clinical manifestations in the Finnish family with the R298G mutation and the patients with the R298Q mutation. The clinical observations in patients from the three families in this study are summarized in Table 1. These features are scored against the typical phenotypic symptoms of each of the six syndromes that are associated with p63 mutations: EEC syndrome, AEC syndrome, Rapp-Hodgkin syndrome, LMS, ADULT syndrome, and SHFM. It appears that the phenotype of patients from the Finnish family is most similar to the ADULT syndrome phenotype. However, also some differences can be noted, such as the lack of neurodermatitic signs and the SCC in one individual. The same is true for the Italian and the Danish families with the R298Q mutation. Patients from the Italian family have the typical hallmarks of ADULT syndrome, but differ because of the normal hair phenotype, conjunctivitis, photosensitivity and albinoid appearance. The Danish family differs from the original ADULT family because of the lack of hypohidrosis, but presents the excessive freckling.

\section{Discussion}

We have identified a p63 mutation in three unrelated families affecting arginine 298 in the DNA binding domain of p63. One mutation, R298G, was not detected before, but we have demonstrated that it is functionally equivalent to the R298Q mutation in two families diagnosed as ADULT syndrome, and to the second and third family reported here. $^{2,6,7}$ Comparison of all 16 patients with a R298 mutation allows a better delineation of the ADULT syndrome phenotype.

Hand and/or foot malformations are observed in all ADULT patients except one (15/16). Ectodermal dysplasia is present in all individuals with R298 mutation and always affects the nails, teeth and skin. Nails are dysplastic, discoloured, brittle and/or have pits. Hypodontia, oligodontia and/or small teeth are a common combination, in addition to loss of permanent teeth in young adulthood. The skin is mostly dry and thin, sometimes with neurodermatitic signs. Also, frequently observed are defects of the mammary glands, hair and lacrimal ducts. Mammary gland and/or nipple hypoplasia is present in 12 out of 16 patients, including males. Some ADULT patients show signs that may be family-specific traits. For example, photosensitivity and hypopigmentation are only seen in the Italian family. Also, the extensive freckling, which stands as one of the typical hallmarks of ADULT syndrome, is observed in the original ADULT syndrome family, in the Danish family, and a very mild version (freckling on cheeks) is reported in a sporadic ADULT syndrome case. ${ }^{2,7}$
Even in the original ADULT syndrome family the freckling is not a consistent feature. Therefore, it is possible that other genetic factors contribute to this part of the phenotype either as a major gene or as a modifier. Finally, oral SCC was seen in one of the patients, but its relation to the p63 R298G mutation is obscure (see below).

In conclusion, ADULT syndrome is the most likely clinical diagnosis in a patient with ectrodactyly, mammary gland hypoplasia, neurodermatitic signs, prominent ectodermal dysplasia and normal lip and palate. This specific combination of clinical features is a-typical for EEC syndrome. However, the high degree of clinical variability in EEC syndrome may confuse a correct clinopathological diagnosis, especially for isolated patients. Presence of clefting or absence of mammary gland involvement is indicative of EEC syndrome. LMS is clinically very similar to ADULT syndrome, but differs by much milder ectodermal involvement. Absence of neurodermatitic signs such as freckling or hypopigmentation does not preclude a diagnosis of ADULT syndrome.

Having defined the common features associated with mutations at R298, it is of interest to compare these with the single ADULT syndrome patient with another mutation, N6H (Table 1). ${ }^{5}$ The N6H patient has ADULT signs such as limb malformation, nipple hypoplasia and lacrimal duct obstruction, nail dysplasia and hypodontia. However, she does not have hair abnormalities, alopecia or skin involvement. This patient has freckling, but this was absent in two of the five families with R298 mutations. We believe that freckling may not be a discriminating factor in ADULT syndrome characteristics. Altogether $\mathrm{N6H}$ mutation causes a syndrome, which equally resembles ADULT syndrome and LMS. Thus, this patient cannot be confidently classified as either ADULT or LMS. The location of the N6H mutation, in the beginning of deltaNp63, could indicate LMS syndrome, since the G76W mutation is only 15 amino acids downstream in deltaNp63. (The numbering of these mutations is based on different p63 isoforms)

We have shown the functional consequences of the R298 mutation in vitro. This mutation induces transactivation activity for a deltaNp63gamma isoform, which usually does not have this capacity. Although the mechanism of the gain-of-function effect is still unclear, this observation has led to the identification of a putative second transactivation domain uniquely present in the deltaN-isoforms. ${ }^{6}$ Here, we have shown that the gain-of-function effect is not specific to R298Q. The R298G substitution has the same functional effect. This result indicates that the mechanism of these mutations depends on the removal of arginine 298 , and consequently the disruption of p63 properties that are mediated through this amino acid, and not so much by novel properties that are brought about by the substituted amino acid.

As p63 is a family member of the well-known tumoursuppressor gene p53, it was suspected to have a role in 
Table 1 ADULT syndrome characteristics caused by R298 mutations are compared to other allelic syndrome features ${ }^{\mathrm{a}}$ and other reported ADULT syndrome features (N6H and R298Q) $)^{2,4,5,7}$

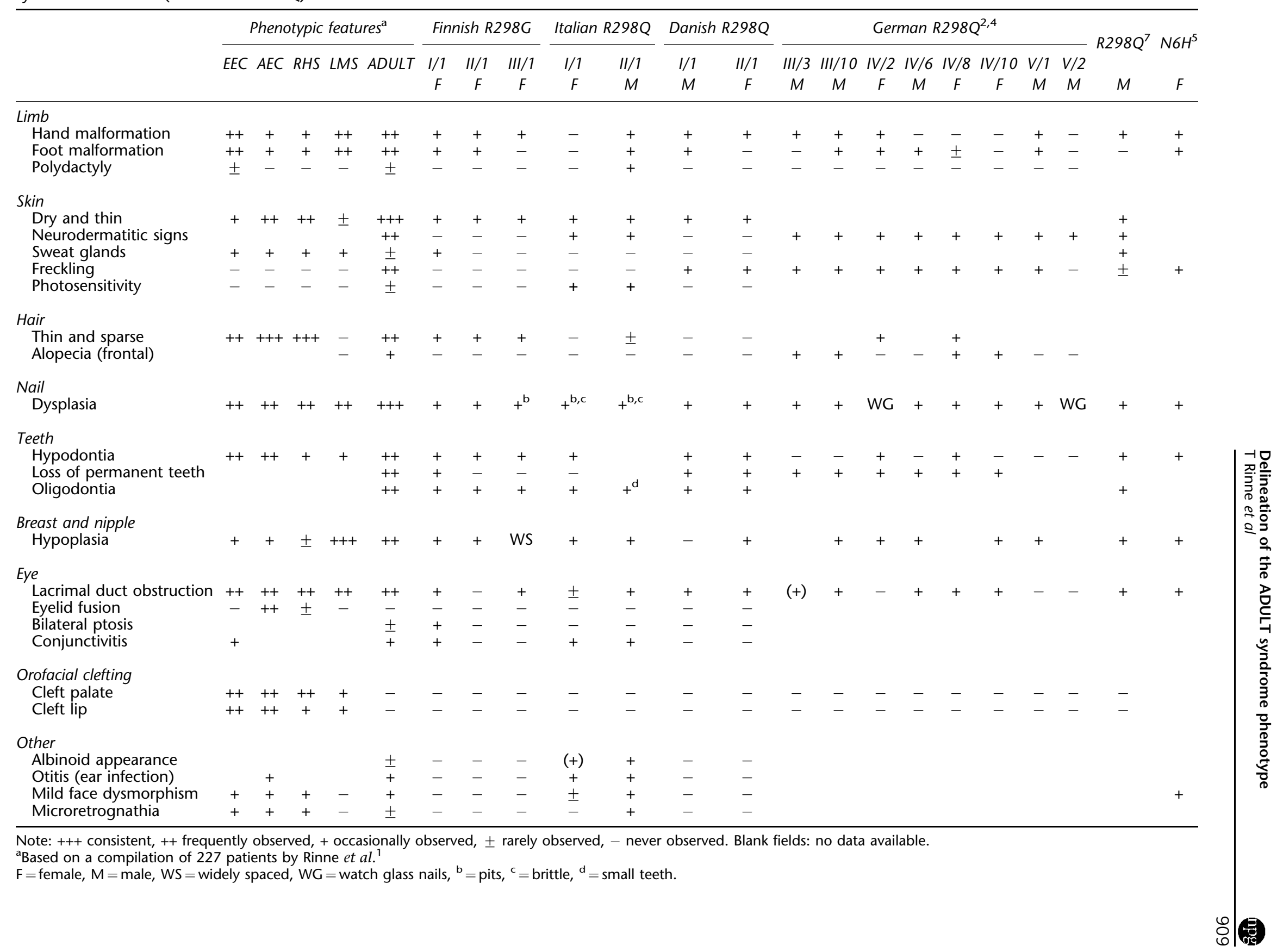


cancer development from its discovery. There are several reports of strong p63 accumulation in basal cell carcinomas and SCCs. ${ }^{11-15}$ Also, the undifferentiated SCC of the mouth of the ADULT patient (R298G) described in this article showed strong p63 accumulation (Figure 3). A clearcell-renal carcinoma was reported for the maternal grandfather of the Italian family, but we were unable to establish whether he was a carrier of the R298Q mutation. So far only one EEC patient with p63 mutation (D312G) has been described to have a cancer, a malignant B-cell lymphoma. ${ }^{16}$ These are the only reported descriptions of human p63 patients developing cancer, suggesting that individuals with a germline p63 mutation have little or no increased risk to develop cancer. A recent study with heterozygous p53, p63 and p73 knockout mice studies reported that the lifespan for each genotype was reduced and incidence of the tumours was increased. ${ }^{17}$ Loss of heterozygosity of each of the p53 family members was observed in these tumours. Interestingly, SCC was observed in 10\% of heterozygous p63 mutant mice. Complete loss of heterozygosity in this cancer type further suggested a tumour-suppressor role for p63. This observation is in sharp contrast to previous suggestions that p63 acts as an oncogene and accumulates in tumours, notably in SCCs as in the present patient with an R298Q mutation. ${ }^{11-15}$ An oncogenic activity for p63 would also be in line with the gain-of-function activity that is brought about by the R298 amino-acid substitutions. Nevertheless, since SCCs are common (7-11\% lifetime risk ${ }^{18}$ ) we cannot conclude that there is a causal relationship between the SCC and the p63 mutation in the present ADULT syndrome patient.

\section{Acknowledgements}

We thank all the patients for their participation in this study. This work was supported by ZonMW (901-02-232) and European Union Sixth Framework programme EpiStem project (LSHB-CT-2005019067). Wilhelm Johannsen Centre for Functional Genome Research was established by the National Danish Research Foundation.

\section{References}

1 Rinne T, Hamel BC, van Bokhoven H, Brunner HG: Pattern of p63 mutations and their phenotypes - update. Am J Med Genet A 2006, in press.
2 Propping P, Zerres K: ADULT-syndrome: an autosomal-dominant disorder with pigment anomalies, ectrodactyly, nail dysplasia, and hypodontia. Am J Med Genet 1993; 45: 642-648.

3 van Bokhoven $\mathrm{H}$, Jung $\mathrm{M}$, Smits $\mathrm{AP}$ et al: Limb mammary syndrome: a new genetic disorder with mammary hypoplasia, ectrodactyly, and other Hand/Foot anomalies maps to human chromosome 3q27. Am J Hum Genet 1999; 64: 538-546.

4 Propping P, Friedl W, Wienker TF, Uhlhaas S, Zerres K: ADULT syndrome allelic to limb mammary syndrome (LMS)? Am J Med Genet 2000; 90: 179-182.

5 Amiel J, Bougeard G, Francannet C et al: TP63 gene mutation in ADULT syndrome. Eur J Hum Genet 2001; 9: 642-645.

6 Duijf PH, Vanmolkot KR, Propping P et al: Gain-of-function mutation in ADULT syndrome reveals the presence of a second transactivation domain in p63. Hum Mol Genet 2002; 11: 799-804.

7 Chan I, Harper JI, Mellerio JE, McGrath JA: ADULT ectodermal dysplasia syndrome resulting from the missense mutation R298Q in the p63 gene. Clin Exp Dermatol 2004; 29: 669-672.

8 Celli J, Duijf P, Hamel BC et al: Heterozygous germline mutations in the p53 homolog p63 are the cause of EEC syndrome. Cell 1999; 99: 143-153.

9 Fomenkov A, Huang YP, Topaloglu O et al: P63 alpha mutations lead to aberrant splicing of keratinocyte growth factor receptor in the Hay-Wells syndrome. J Biol Chem 2003; 278: 23906-23914.

10 van Bokhoven H, Brunner HG: Splitting p63. Am J Hum Genet 2002; 71: 1-13.

11 Parsa R, Yang A, McKeon F, Green H: Association of p63 with proliferative potential in normal and neoplastic human keratinocytes. J Invest Dermatol 1999; 113: 1099-1105.

12 Hibi K, Trink B, Patturajan $\mathrm{M}$ et al: AIS is an oncogene amplified in squamous cell carcinoma. Proc Natl Acad Sci USA 2000; 97: $5462-5467$.

13 Nylander K, Coates PJ, Hall PA: Characterization of the expression pattern of p63 alpha and delta Np63 alpha in benign and malignant oral epithelial lesions. Int $J$ Cancer 2000; 87: $368-372$.

14 Di Como CJ, Urist MJ, Babayan I et al: p63 expression profiles in human normal and tumor tissues. Clin Cancer Res 2002; 8: 494-501.

15 Reis-Filho JS, Torio B, Albergaria A, Schmitt FC: p63 expression in normal skin and usual cutaneous carcinomas. I Cutan Pathol 2002; 29: 517-523.

16 Akahoshi K, Sakazume S, Kosaki K, Ohashi H, Fukushima Y: EEC syndrome type 3 with a heterozygous germline mutation in the P63 gene and B cell lymphoma. Am J Med Genet A 2003; 120: $370-373$.

17 Flores ER, Sengupta S, Miller JB et al: Tumor predisposition in mice mutant for p63 and p73: evidence for broader tumor suppressor functions for the p53 family. Cancer Cell 2005; 7: $363-373$.

18 Miller DL, Weinstock MA: Nonmelanoma skin cancer in the United States: incidence. I Am Acad Dermatol 1994; 30: $774-778$. 